\title{
Electron Photodetachment from Halide Ions in Solution: Excited-State Dynamics in the Polarization Well
}

\author{
Frederick H. Long, ${ }^{\dagger}$ Xuelong Shi, ${ }^{\ddagger}$ Hong Lu, and Kenneth B. Eisenthal ${ }^{*}$ \\ Department of Chemistry, Columbia University, New York, New York 10027
}

Received: September 27, 1993; In Final Form: April 12, $1994^{\circ}$

\begin{abstract}
The electronic relaxation and photodetachment dynamics of an electron originating from an aqueous iodide ion have been studied using femtosecond absorption spectroscopy. The results indicate the importance of an upper excited charge transfer to solvent (CTTS) state, of odd parity, in the potential well of the solvated ion. The upper excited CTTS state, which is accessed by two-photon excitation, relaxes in $<50 \mathrm{fs}$ to the lowest excited CTTS, which in turn relaxes to the ground state of the I-in $80 \mathrm{fs}$. It is found that most of the solvated electrons are produced by a three-photon excitation to a photoionized state, rather than from the CTTS excitation.
\end{abstract}

\section{Introduction}

The subject of electrons in fluids is of contemporary interest to a wide variety of chemists and physicists. ${ }^{1-22}$ In recent years there have been a number of studies of electrons in neat water using femtosecond absorption spectroscopic methods ${ }^{1} 1^{5}$ and theoretical and computer simulation methods. ${ }^{7-13}$ The model that has emerged from both the experimental and theoretical work is one in which the dynamics of electron solvation is described as an excited-state relaxation process. ${ }^{1,5,10-13}$

The related subjects of aqueous ions and electrolyte solutions are also areas of longstanding fundamental interest in physical chemistry. Many simple anions in polar solution such as halides have large, broad absorption spectra in the UV. The transitions correspond to the excitation of an electron of the anion in the polarization well created by the solvent molecules oriented around the anion. The spectra are commonly referred to as chargetransfer-to-solvent or CTTS spectra. ${ }^{15-20}$ In our earlier studies of electron photodetachment in electrolyte solutions, ${ }^{21,22}$ we detected an ultrafast transient visible absorption in both $\mathrm{I}^{-}$and Br- solutions. ${ }^{22}$ We interpreted this transient as an excited state of the electron in the orientational polarization well formed by the waters organized around the solvated ion. In this paper, new experimental studies of electronic relaxation, electron photodetachment, and solvation of iodide anions in aqueous solution are presented and are compared with recent quantum mechanical simulations. ${ }^{13}$

\section{Experimental Section}

The experiments performed were transient absorption measurements using an amplified colliding-pulse-mode-locked dye laser operating at $10 \mathrm{~Hz}, 625 \mathrm{~nm}$, and $300 \mu \mathrm{J} /$ pulse. The 312.5 $\mathrm{nm}$ UV pump pulses were generated by frequency doubling the fundamental beam with a $200-\mu \mathrm{m} \beta$-barium borate (BBO) crystal. The probe beam was either a small fraction of the remaining fundamental or part of a white light continuum that was generated by focusing the fundamental beam into a $1-\mathrm{cm}$-path length $\mathrm{D}_{2} \mathrm{O}$ cell. Different wavelengths were selected by using the appropriate broad-band interference filter. From the autocorrelation and cross-correlation measurements, the fundamental pulse width was found to be $80-\mathrm{fs}$ fwhm, the UV pulse was about $150 \mathrm{fs}$, and the probe pulse from continuum generation was $170-\mathrm{fs}$ fwhm. In order to minimize group velocity dispersion between the pump and the probe, a $200-\mu \mathrm{m}$ cuvette was used in the experiments.

† MS C345, Los Alamos National Laboratories, Los Alamos, NM 87545. t Department of Chemistry, University of Michigan, Ann Arbor, MI 48109.

1 Department of Chemistry, Northwestern University, Evanston, IL 60208.

- To whom correspondence should be addressed.

- Abstract published in Advance ACS Abstracts, June 15, 1994.
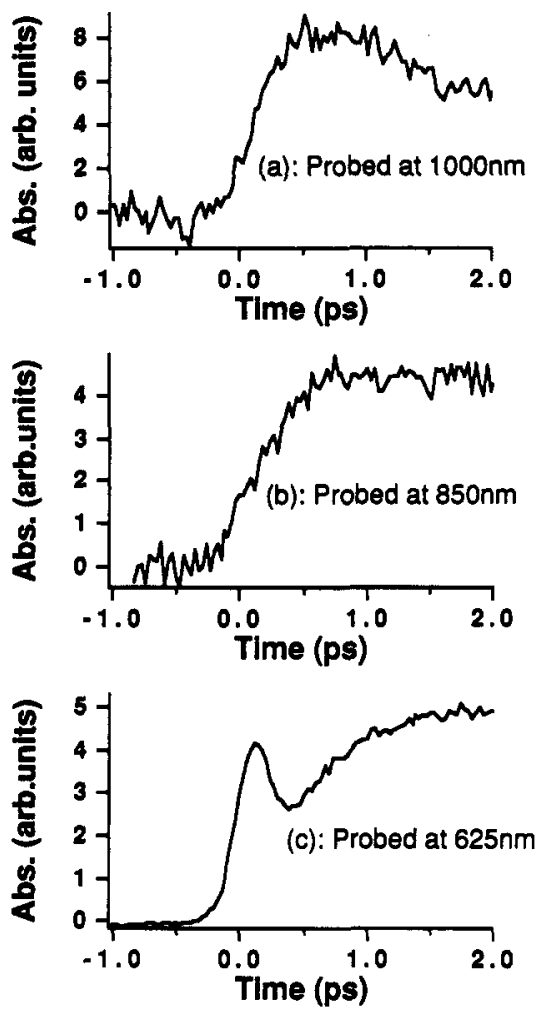

Figure 1. Dynamics of the photodetached electron observed at three different probe wavelengths (1.0 M KI solution in water): (a) $\lambda_{\text {probe }}=$ $1000 \mathrm{~nm}$, (b) $\lambda_{\text {probe }}=850 \mathrm{~nm}$, (c) $\lambda_{\text {probe }}=625 \mathrm{~nm}$. Except for the 625-nm probe, the dynamics are similar to those observed for neat water.

The water used was double distilled, and the $\mathrm{NaI}$ or $\mathrm{KI}$ was the highest grade purity from Aldrich.

\section{Results and Discussion}

Dynamics of the Wet Electron. In Figure 1, the observed dynamics of the aqueous electron originating from the iodide ion are shown. In order to eliminate the small background signal from the water molecules, the UV pump beam was defocused. Data taken with a $1000-\mathrm{nm}$ IR probe pulse is qualitatively similar to but quantitatively different from that seen in neat water (Figure 1a). The rise of the signal corresponds to the formation of the wet electron and the decay to the formation of the fully solvated electron. The formation of the solvated electron appears as a decay in the IR because the wet electron absorbs more strongly than the solvated electron in this region. In Figure 1b, the photodetachment dynamics probed at $850 \mathrm{~nm}$ are shown. After the formation of the wet electron, the absorption appears to be 
nearly constant. This behavior is characteristic of an isosbestic point. The significance of this observation will be discussed in more detail later. In Figure 1c, a $625-\mathrm{nm}$ probe was used, and a dramatic departure from the previous experiments in neat water is seen in the rapid rise and decay at early times. The signal appears before the formation time of the wet electron. This species is the trapped or CTTS electron. This early time signal corresponds to the electron in the potential well of the initially solvated anion; i.e., we are observing the absorption from an excited electronic state in the CTTS well. ${ }^{22}$ It is important to note that the subsequent solvation dynamics at $625 \mathrm{~nm}$ are qualitatively similar to that seen in neat water and corresponds to solvation of electrons not in the polarization well of the initial ion.

Electron solvation dynamics in neat liquid water are characterized by the observation of two species, the wet and the solvated electron. The wet electron is the IR absorbing precursor of the solvated electron. The two-state character of the solvation dynamics was confirmed by the discovery of an isosbestic wavelength in the electron solvation dynamics. ${ }^{1}$ At the isosbestic wavelength, the absorption coefficient of the wet and the solvated electron are equal, and thus the wet and the solvated electron are indistinguishable in their optical absorption. We used a kinetic model for the analysis of the electron solvation dynamics in neat water.'

$$
\mathrm{e}_{\text {quasi-free }} \stackrel{k_{1}}{\rightarrow} \mathrm{e}_{\text {wet }} \stackrel{k_{2}}{\rightarrow} \mathrm{e}_{\text {solvated }}
$$

We will use a similar kinetic model for the electron solvation dynamics in the electrolyte solutions. The electrons generated in these solutions, however, come from the multiphoton electron photodetachment of the $\mathrm{I}^{-}$and not by photoionization of the water molecules. At the laser powers used in these experiments only a negligible electron population is obtained from a neat water system. From our wavelength-dependent data, we can obtain information about the formation, decay, and absorption spectrum of the wet electron. From the rise of the signal at $850 \mathrm{~nm}$, we find that the wet electron formation time $\left(1 / k_{1}\right)$ is $400 \pm 40$ fs. This is slightly longer than the value obtained for neat water, 300 $\pm 40 \mathrm{fs}$. From the data at 625 and $1000 \mathrm{~nm}$, we find that the time for the wet electron to decay into the solvated electron (1/ $k_{2}$ ) is $600 \pm 40 \mathrm{fs}$, which is close to the value of $1 / k_{2}$ for neat water, $540 \pm 40 \mathrm{fs}$. The small but real change in the formation kinetics of the wet electron could be due to the perturbation of the water structure by the anions and cations in the neighborhood of the photoejected electron, which slows the reorientation of the water molecules in their approach to the equilibrium geometry of the solvated electron. If the electrolyte was the origin of the change, then we would expect that the dynamics would depend on the electrolyte concentration. We found that the kinetics remains the same in the concentration range studied, $0.25-2.0 \mathrm{M}$, which, though somewhat surprising, may not be conclusive since the range of interionic separations is not very large. Another possibility is that the photoejected electron is sufficiently close to the parent iodide ion to be perturbed by the remaining iodine atom and its surrounding water molecules. The water molecules reorganize from the initial geometry determined by the anion to a geometry influenced by the newly formed iodine atom and the photoejected electron. The longer solvation times could be due to the increased nuclear motion required for these water molecules to move from their initial polarized state to the equilibrium solvated electron geometry.

In our search for an isosbestic wavelength, we scanned the solvation kinetics as a function of probe wavelength (Figure 1). We found at $850 \pm 30 \mathrm{~nm}$ that the signal rose monotonically and leveled off, which indicates an isosbestic wavelength. This is close to the value we obtained in neat water where the isosbestic wavelength was found to be $820 \pm 30 \mathrm{~nm}$. It is of interest to note that there is no significant geminate recombination of the electron
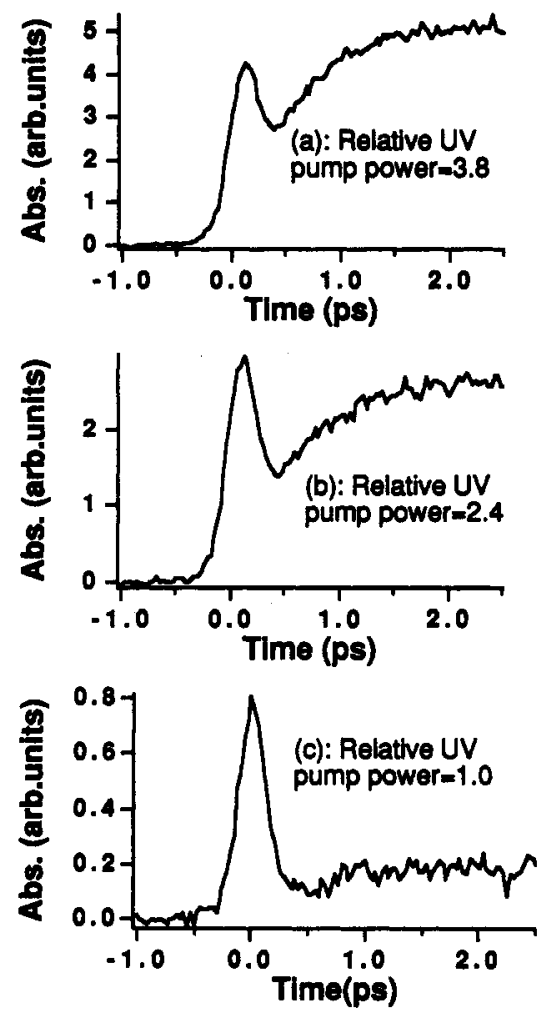

Figure 2. Intensity dependence of the CTTS signal (a) UV $=3.8$, (b) $\mathrm{UV}=2.4$, (c) $\mathrm{UV}=1.0(625-\mathrm{nm}$ probe). The dramatic intensity dependence of the signal suggests that the trapped (CTTS) electron is produced by a two-photon process and the solvated electron is produced by a three-photon process.

with the iodine atom on the 2-ps time scale. The absence of an early time geminate recombination of the solvated electron is a direct consequence of the lack of a strong interaction between the solvated electron and the neutral atom, unlike the neat water where the solvated electron and the $\mathrm{H}_{2} \mathrm{O}^{+}$ion, more likely the daughter $\mathrm{H}_{3} \mathrm{O}^{+}$ion (and $\mathrm{OH}$ ), interact strongly by the Coulomb force.

Dynamics of the CTTS Electron. We now consider the CTTS electron dynamics. The transient CTTS electron signal is observed between 700 and $450 \mathrm{~nm}$ with an absorption maximum at 550 nm. ${ }^{22}$ With a 625-nm probe, the formation time was found to be $\leq 50 \mathrm{fs}$, which is instrument limited, and the decay time was found to be $80 \pm 30 \mathrm{fs}$. It was found that the kinetics of the transient CTTS electron are wavelength dependent. With a 550$\mathrm{nm}$ probe, the decay kinetics are slower than that observed with a 600 -nm probe, which suggests as a possibility that the adiabatic relaxation on the potential surface of the lowest excited CTTS state is contributing to the observed transient absorption and consequently the observed wavelength-dependent kinetics.

The transient CTTS spectra should be general to halide ions in polar fluids. For example, we observed the transient CTTS electron for solutions of $\mathrm{I}^{-}$in methanol as well as in water. ${ }^{23}$ In addition, we have observed the transient CTTS electron originating from the bromine anion in water. Using a $450-\mathrm{nm}$ probe, it appears that the transient CTTS state absorption in the polarization well of the $\mathrm{Br}$ - ion is blue-shifted with respect to the $\mathrm{I}^{-22}$. The blue shift of the transient CTTS electron absorption in the $\mathrm{Br}$ solution is likely due to the decrease in the ion radius. A decreased ion radius will increase the separation between the energy levels of the CTTS polarization well. Attempts to observe the transient CTTS state absorption for $\mathrm{Cl}^{-}$and $\mathrm{OH}^{-}$at $400 \mathrm{~nm}$ were unsuccessful; it is likely their absorptions lie further in the UV.

Pump Intensity Dependence of the Dymamics. A dramatic intensity dependence is observed in the dynamics of the photodetached electron. See Figure 2a-c. At relatively high pump intensities (Figure 2a, $U V=3.8$ ), the relative height of the solvated 


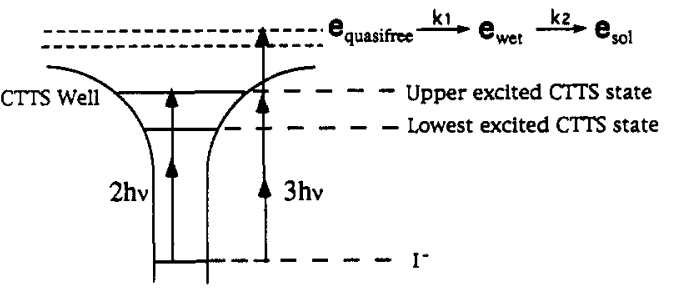

Figure 3. Energy diagram showing the CTTS potential well, the twoand three-photon excitation processes observed in our experiments, and the transient CTTS absorption from the lowest CTTS state.

electron absorption signal obtained from the longer time plateau region is larger than the transient CTTS signal seen as a peak at earlier times. At lower pump fields (Figure $2 c, U V=1.0$ ), the opposite trend is observed; the transient CTTS signal is much larger than the solvated electron signal. In the arbitrary units used for UV intensity, $U V=1.0$ is approximately $I \approx 10^{11} \mathrm{~W} / \mathrm{cm}^{2}$. In fact, we can almost eliminate the solvated electron signal at low UV pump intensities. The same intensity dependence is also observed with a $550-$ or $600-\mathrm{nm}$ probe. This phenomena can be understood if the CTTS state is produced by a two-photon process whereas the solvated electron is predominantly produced by a three-photon or a $2+1$ excitation process. Therefore, at low UV intensities the transient CTTS signal dominates, and at high UV intensities the three-photon mechanism is comparable to the twophoton mechanism. The pump photon has $4.0 \mathrm{eV}$ of energy; therefore, a two-photon transition will lie above the first excitedstate in the CTTS well (Figure 3). A similar competition between two- and three-photon processes has been observed in our neat water experiments. ${ }^{2}$ It appears that in our experiments the vast majority of solvated electrons are produced by the three-photon excitation mechanism. The relaxation of most of the CTTS electrons to the ground state of the anion has also been observed in simulations..$^{13}$ In addition, steady-state measurements by Jortner et al., at neutral $\mathrm{pH}$, found a low $5 \%$ quantum yield of solvated electrons from excitation into the first excited state. ${ }^{17}$ The observed UV intensity dependence can be compared with estimates of the polarization well depth. From the Franck model, the well depth can be estimated to be about $7.5 \mathrm{eV} .{ }^{15,19}$ This is not much different than the 8.0-eV, two-photon excitation energy, indicating that the potential is at least $0.4 \mathrm{eV}$ deeper than the Platzmann-Franck prediction. The three-photon process at 12 eV clearly has sufficient energy to photodetach the electron and thus produce a quasi-free electron.

We can estimate the value of $\sigma_{2}$, which is the two-photon cross section for the 8.0-eV excitation of an upper CTTS state for Iin aqueous solution, by using the known value of $\sigma_{2}=9 \times 10^{-52}$ $\mathrm{cm}^{4} \mathrm{~s}^{2}$, obtained by Nikogosan, for neat water at $316 \mathrm{~nm} .{ }^{24}$ The transient CTTS signal from iodide, at $1.0 \mathrm{M}$ concentration, is typically 10 times stronger than the signal from neat water. Therefore, the cross section is estimated to be about 500 times larger for the iodide ion based on the molarity of water as $55 \mathrm{M}$. This implies a cross section of $\approx 4 \times 10^{-49} \mathrm{~cm}^{4} \mathrm{~s}$. On the basis of the expectation that a transition to a continuum final state would not be this strong, we conclude that there is an upper excited CTTS state inside the polarization well of the $\mathrm{I}^{-}$anion at $8.0 \mathrm{eV}$.

Additional evidence for the role of an upper excited CTTS state comes from a consideration of selection rules. The groundstate absorption is a strongly allowed one-photon transition from the ground p-like state to an s-like state for the electron. ${ }^{15-19}$ Selection rules for two-photon transitions require that parity not change; therefore, the two-photon excitation would place the electron in a p-like state. The existence of two excitation pathways and the odd parity excited CTTS state has been seen in the simulations of Sheu and Rossky. ${ }^{13}$

We have also examined the intensity dependence of the signal at $850 \mathrm{~nm}$, where no signal from the CTTS electron is observed.

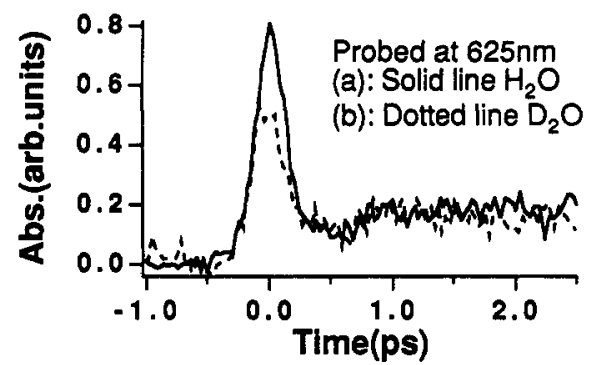

Figure 4. Isotope dependence of CTTS dynamics probed at $625 \mathrm{~nm}$ : $\mathrm{H}_{2} \mathrm{O}$, solid line; $\mathrm{D}_{2} \mathrm{O}$, dotted line (1.0 M KI aqueous solution). Note the fraction of trapped electrons is significantly larger in $\mathrm{H}_{2} \mathrm{O}$.

It is found that the formation rate of the wet electron is not intensity dependent. This is consistent with our conclusion that the solvated electrons we are observing are produced primarily by a three-photon ionization of the iodide and not by a twophoton excitation of a CTTS state followed by a transition to the ground or excited state of the solvated electron. If there was a mixture of different generation mechanisms for wet electrons, then one would expect $k_{1}$, the wet electron formation time, to be intensity dependent.

Isotope Effect Studies of the Dynamics. Measurements of the isotope dependence of the wet electron formation rate have also been performed. The isotope effect is small and close to our experimental limits. The wet electron formation rate in the $\mathrm{D}_{2} \mathrm{O}$ iodide solution was found to be $480 \pm 40 \mathrm{fs}$ vs $400 \pm 40 \mathrm{fs}$ in the $\mathrm{H}_{2} \mathrm{O}$ iodide solution. The solvated electron formation time in the $\mathrm{D}_{2} \mathrm{O}$ iodide solution was found to be $680 \pm 40$ fs vs $600 \pm 40$ fs in the $\mathrm{H}_{2} \mathrm{O}$ iodide solution. This isotope effect is similar in magnitude to that found for neat water, i.e., roughly a $10-15 \%$ decrease in the kinetics for the deuterated solvent.

A dramatic isotope effect is observed, however, in the formation and decay dynamics of the lowest excited CTTS state (Figure 4). The CTTS absorption amplitude was found to be larger for $\mathrm{H}_{2} \mathrm{O}$ than $\mathrm{D}_{2} \mathrm{O}$ at all pump intensities and probe wavelengths. Thus, we conclude from this that the observed isotope effect is not related to the two- vs three-photon process discussed earlier. Because one would not expect strong spectral changes upon isotope substitution to be responsible for the different absorption in $\mathrm{H}_{2} \mathrm{O}$ and $\mathrm{D}_{2} \mathrm{O}$ shown in Figure 4, we attribute this decrease in the transient CTTS signal to a change in the formation and decay kinetics of the lowest excited CTTS state.

\section{Summary and Conclusions}

The dynamics of electronic relaxation following photoexcitation of $\mathrm{I}^{-}$in aqueous solution has been investigated using femtosecond laser methods. We have observed ultrafast electronic relaxation, following two-photon $\left(2 \omega_{\text {pump }}=8.0 \mathrm{eV}\right)$ photoexcitation to an upper CTTS (charge transfer to solvent) state of the potential well created by the polarization of the water molecules surrounding the I-. The relaxation time of the upper CTTS state to the lowest CTTS state is less than $50 \mathrm{fs}$, which is an instrument-limited result in our experiment. The electron in the lowest CTTS state then relaxes in $80 \pm 30 \mathrm{fs}$ to the ground state of the $I^{-}$in the orientational polarization well of the water molecules. The dynamics were monitored by measurements of the time-dependent absorption from the lowest CTTS state. We also found that the yield of solvated electrons from two-photon excitation to an upper CTTS state was small. The primary source of the solvated electrons is a three-photon excitation of the $\mathrm{I}^{-}$to a photoionized state at $12 \mathrm{eV}$.

Acknowledgment. The authors thank the The Division of Chemical Science of the Department of Energy for their support and the National Science Foundation and the Air Force Office of Scientific Research for their equipment support. F.H.L. thanks IBM for a predoctoral fellowship. 


\section{References and Notes}

(1) Long, F. H.; Lu, H.; Eisenthal, K. B. Chem. Phys. Lett. 1989, 160 , 464. Long, F. H.; Lu, H.; Eisenthal, K. B. Phys. Rev. Lett. 1990, 64, 1469. Lu, H.; Long, F. H.; Eisenthal, K. B. Opt. Soc. Am. B 1990, 7, 1513. 185,47

(2) Long, F. H.; Lu, H.; Shi, X.; Eisenthal, K. B. Chem. Phys. Lett. 1991,

(3) Migus, A.; Gaudel, Y.; Martin, J. L.; Antonetti, A. Phys. Rev. Lett. $1987,58,1559$

(4) Gaudel, Y.; Pommeret, S.; Migus, A.; Yamada, N.; Antonetti, A.J. Am. Chem. Soc. 1990, 112, 2925. Gaudel, Y.; Pommeret, S.; Migus, A.; Antonetti, A. J. Phys. Chem. 1991, 95, 533.

(5) Alfano, J. C.; Walhout, P. K.; Kimura, Y.; Barbara, P. F. J. Chem. Phys. 1993, 98, 5996.

(6) Padowitz, D. F.; Merry, W. R.; Jordan, R. E.; Harris, C. B. Phys. Rev. Lett. 1992, 69, 3583.

(7) Schnitker, J.; Motakabbir, K.; Rossky, P. J.; Friesner, R. Phys. Rev. Lett. $1988,60,456$. 4277.

(8) Wallquist, A.; Martyna, G.; Berne, B. J. J. Phys. Chem. 1988, 92,

(9) Rossky, P. J.; Schnitker, J. J. Phys. Chem. 1989, 92, 4277.

(10) Webster, F.; Schnitker, J.; Rossky, P. J.; Freisner, R. Phys. Rev. Lett. 1991, 66, 3172. Murphery, T. H.; Rossky, P. J. J. Chem. Phys. 1993, $99,515$. sen: Keszei, E. et al., J. Chem. Phys. 1993, 99, 2004.
(11) Space, B.; Coker, D. F. J. Chem. Phys. 1991, 94, 1976.

(12) Neria, E.; Nitzan, A.; Barnett, R. N.; Landman, U. Phys. Rev. Lett. $1991,67,1011$.

(13) Sheu, W.-S.; Rossky, P. J. Chem. Phys. Lett. 1993, 213, 233.

(14) Messmer, M. C.; Simon, J. D. J. Phys. Chem. 1990, 94, 1220.

(15) Franck, J.; Scheibe, G. Z. Phys. Chem. 1928, Al39, 22.

(16) Grossweiner, L. I.; Matheson, M. S. J. Phys. Chem. 1957, 61, 1089. Matheson, M. S.; Mulac, W. A.; Rabani, J. J. Phys. Chem. 1963, 67, 261.

(17) Jortner, J.; Ottolenghi, M.; Stein, G. J. Phys. Chem. 1962, 66, 2029. Jortner, J.; Ottolenghi, M.; Stein, G. J. Phys. Chem. 1962, 66, 2037.

(18) Dainton, F. S.; Logan, S. R. Proc. R. Soc. London 1965, A287, 281.

(19) For a general review see: M. J.; Blandamer, Fox, M. F. Chem. Rev. $1970,70,59$ and references therein.

(20) Iwata, A.; et al. Chem. Phys. Lett. 1993, 207, 137.

(21) Long, F. H.; Lu, H.; Eisenthal, K. B. J. Chem. Phys. 1989, $91,4193$. (22) Long, F. H.; Lu, H.; Shi, X.; Eisenthal, K. B. Chem. Phys. Lett. 1990 , 169,165 .

(23) Unpublished results from this laboratory.

(24) Nikogosyan, D. N.; Oraersky, A. A.; Rupasov, V. I. Chem. Phys. $1983,77,131$.

(25) Eberly, J. H. In Advances in Chemical Physics; Hirshfelder, J. O.; Wyatt, R. E.; Coalson, R. D., Eds.; Wiley: New York, 1989; Vol. 73. 